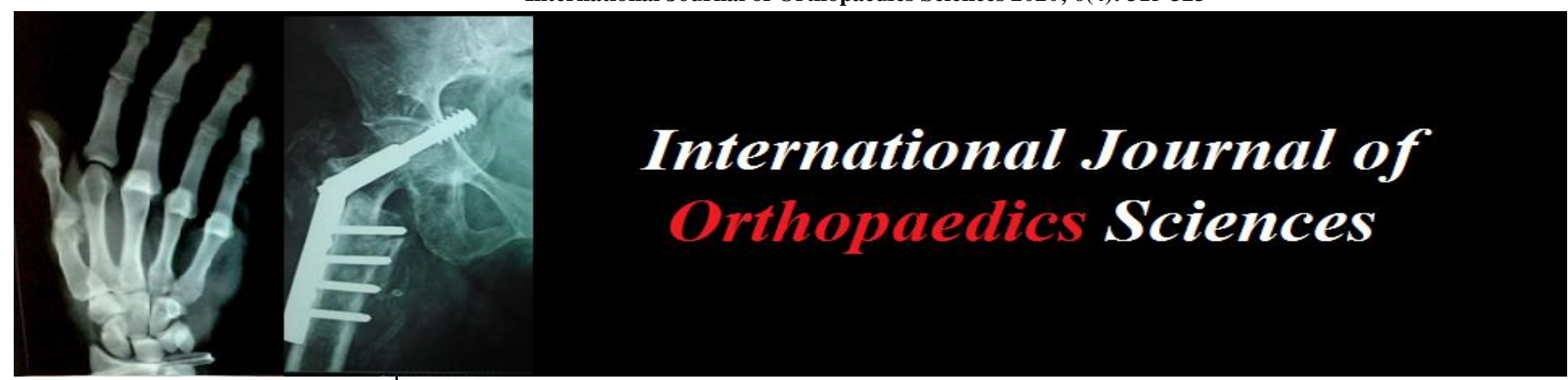

E-ISSN: 2395-1958

P-ISSN: 2706-6630

IJOS 2020; 6(4): 315-323

(C) 2020 IJOS

www.orthopaper.com

Received: 21-07-2020

Accepted: 28-08-2020

Dr. G Vasudevan

Post graduate, Department of

Orthopaedics, Rajah Muthiah

Medical College, Chidambaram,

Tamil Nadu, India

Dr. Dhanpal Singh

Professor, Department of

Orthopaedics, Rajah Muthiah

Medical College, Chidambaram,

Tamil Nadu, India

Dr. A Manikandarajan

Assistant Professor, Department of Orthopaedics, Rajah Muthiah

Medical College, Chidambaram,

Tamil Nadu, India
Corresponding Author: Dr. Dhanpal Singh

Professor, Department of

Orthopaedics, Rajah Muthiah

Medical College, Chidambaram,

Tamil Nadu, India

\section{Retrospective and prospective outcome analysis of segmental fractures of tibia treated with intramedullary nailing}

\author{
Dr. G Vasudevan, Dr. Dhanpal Singh and Dr. A Manikandarajan
}

DOI: $\underline{\text { https://doi.org/10.22271/ortho.2020.v6.i4e.2356 }}$

Abstract

Introduction: Segmental tibial fractures are complex injuries associated with significant soft tissue damage that are difficult to treat. Aim of the study was to evaluate the Functional and Radiological outcome following closed interlocking intramedullary nailing for segmental fractures of tibia.

Method: A retrospective and prospective study Conducted in $\mathrm{RMMCH}$, Annamalai University 32 patients (26 males and 6 females) with segmental tibial fractures were included and functional outcome of these fractures were evaluated after interlocking nailing for tibia. In follow-up, we determined the time to union, delayed and nonunion and overall complication rates and functional outcome by Johner and Wruchs criteria. All patients were followed for eighteen months

Results: Majority of the cases union time lies between 25 to 30 weeks. Proximal segment unites faster than distal segment. Post operative complications noted in 14 cases. Open injuries is related with higher complication rate. Timely intervention secondary procedures were done depending on the complications. Two subjects were fully recovered and implant exit was done. The final outcome based on JOHNER AND WRUCH'S criteria were excellent in 26 cases good in 6 cases.

Conclusion: Patient counselling regarding the complex nature of injury and the possible requirement of secondary procedures should be anticipated and addressed. The results of our study suggest that if softtissue handling and restoration of anatomical alignment are successful in the treatment of challenging segmental tibial fractures, IMN will be good treatment method.

Keywords: Segmental tibia fracture, intramedullary interlocking nail, expert tibia nail.

\section{Introduction}

Rising incidence of high velocity trauma due to motor vehicle accidents usually results in fractures of long bones. The tibia is the most commonly fractured long bone in the body. Segmental tibia fractures is defined as a unique fracture type characterized by least two different fracture lines with a completely isolated intercalary osseous fragment. Incidence is about $12-15 \%$ of all tibia fractures. The aim towards treatment for the segmental fracture tibia is union maintaining normal length, normal alignment without rotational deformity, normal joint movements and reduced hospital stay. Current treatment options are locked intramedullary nailing, external fixators, plate osteosyntheses and plaster of paris cast immobilization. Delayed union and nonunion are Commonly seen with segmental tibia fractures when compared to non segmental tibia fractures. The present study has been taken to review the results of segmental fractures of tibia treated with intramedullary interlocking nailing.

The benefits of intramedullary nailing include load sharing, sparing of extra-osseous blood supply and avoidance of soft tissue dissection thereby minimizing postoperative complications.

\section{Aim of the Study}

To evaluate the Functional and Radiological outcome following open / closed interlocking intramedullary nailing for segmental fractures of tibia 


\section{Materials and Methods}

The data for this study was collected from the patient admitted to Rajah Muthiah Medical College and Hospital, Annamalai University, Chidambaram. This study includes the patients those who fulfilled the inclusion and exclusion criteria admitted to Rajah Muthiah Medical College and Hospital during May 2018 to October 2020. Retrospective cases were taken from the record department of RMMCH.

A sum total of 32 cases of segmental tibia cases reported and and studied. Patients will be followed up for a minimum period of 1 year. With each follow up functional and radiological evaluation will be done.

All patients were subjected to a detailed history andclinical examination. Clinical examination was performed including general, systemic, neurovascular and local examination of injured part. Depending on nature of injury relevant radiological examination was done. Anteroposterior and lateral radiograph of knee with leg with ankle were done to diagnose fracture type. Any intra articular extension was clearly noted, if there is doubt then CT scan is taken. Routine preoperative investigation was followed. Open fractures were immediately irrigated, washed and temporarily immobilized with posterior POP above knee slab or calcaneal pin traction with limb elevation and appropriate analgesics and antibiotics. Patients were operated within 3 to 25 days of hospital admission. Approximate length of the nail was measured in the contralateral leg from the tibial tuberosity to most prominent point of medial malleolus. The diameters of medullary canal at isthumus was measured

\section{Inclusion Criteria}

- Closed and open segmental tibia fractures upto grade IIIA Gustilo Anderson type

- $\quad$ Age $>18$ years and $<65$ years

\section{Exclusion Criteria}

- Associated vascular and neurological injury
- Pathological fracture

- Severe systemic illness like active cancer elsewhere in body, chemotherapy, and other medical contraindication for surgery

Isometric quadriceps exercise and toes mobilization was started from second postoperative day. Regular weekly follow up was done to inspect wound condition and other complication for compound fractures and regular 2 week follow up for close fractures and patient discharged after suture removal.

Passive knee mobilization exercise was started when pain and swelling subside. Active range of motion (ROM) exercise was started after 3 days postoperatively in all patients. Serial radiographs were taken at regular interval and was followed up periodically on outpatient basis. Partial weight bearing was started after 21 days, Non weight bearing walking with the help of a walker started after 3 to 5 days and full weight bearing was started after 4 to 8 weeks depending upon the radiological appearance. Clinical and radiological assessment will be done for pain, deformity, shortening, range of motion of knee, ankle, subtalar joints and radiological union. final outcome will be assessed using Johner and Wruh's criteria.

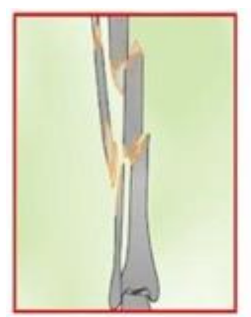

4.2.C2.1
1 segmental fragment

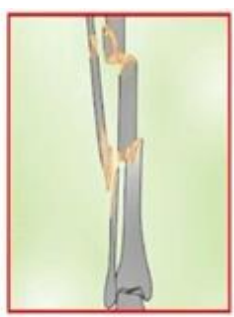

4.2-C2.2 segmental fragment
and additional wedge fragment

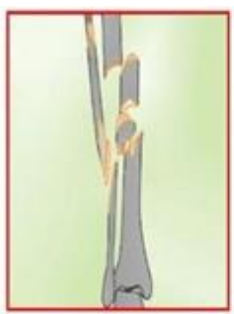

4.2-C2.3

2 segmental fragments
Fig 1: Classification AO classification

Table 1: Gustilo Anderson Classification

\begin{tabular}{|c|c|c|c|c|c|}
\hline & I & II & III-A & III-B & III-C \\
\hline Energy of mechanism & Low & Moderate & High & High & Usually $>10 \mathrm{~cm}$ \\
\hline Wound size & $<1 \mathrm{~cm}$ & $>1 \mathrm{~cm}$ & Usually $>10 \mathrm{~cm}$ & Usually $>10 \mathrm{~cm}$ & Extensive \\
\hline Soft tissue injury & Low & Moderate & Extensive & Variable & Variable \\
\hline Contamination & No & Low & Severe & Severe/Complex & Severe/Complex \\
\hline Conminution/Fracture pattern & No/Simple & Some/Simple & Severe/Complex & Ves & Variable \\
\hline Soft tissue coverage & Yes & Yes & Yes & No, requires reconstructive procedure & No \\
\hline Vascular injury & No & No & No & \multicolumn{2}{c}{ Yesuire reparation } \\
\hline
\end{tabular}

\section{Classification Proposed by Melis et al. in 1981}

They divided segmental tibia fractures into four distinct categories based on fragment fixation with an intramedullary tibial nail construct.

Type I: Defines a segmental fragment between the proximal and middle third of diaphysis of tibia
Type II: Defines a segmental fragment between the middle and distal third of tibial diaphysis.

Type III: Defines a long segmental fragment between the proximal and distal third of tibial diaphysis

Type IV: defines a segmental fragment which is entirely contained in the middle third of the tibial diaphysis.

Table 2: Johner and Wruh's criteria for evaluation of final results

\begin{tabular}{|c|c|c|c|c|c|}
\hline SI. No. & Criteria & Excellent & Good & Fair & Poor \\
\hline 1. & Non-union, infection, Amputations & None & None & None & Yes \\
\hline 2. & Neurovascular disturbances & None & Minimal & Moderate & \\
\hline 3. & Deformity & & & & \\
\hline & Varus/valgus & None & $2-5$ & $6-10$ & $>10$ \\
\hline & Anteversion /Recurvation & $0-5$ & $6-10$ & $11-20$ & $>20$ \\
\hline & Rotation & $0-5$ & $6-10$ & $11-20$ & $>20$ \\
\hline 5. & Shortening & $0-5 \mathrm{~mm}$ & $6-10 \mathrm{~mm}$ & $11-20 \mathrm{~mm}$ & $>20 \mathrm{~mm}$ \\
\hline
\end{tabular}




\begin{tabular}{|c|c|c|c|c|c|}
\hline & Knee & Normal & $>80$ & $>75$ & $<75$ \\
\hline & Ankle & Normal & $>75 \%$ & $>50 \%$ & $<50 \%$ \\
\hline & Subtalar & $>75 \%$ & $>50 \%$ & $<50 \%$ & -- \\
\hline 6. & Pain & None & Occasional & Moderate & Severe \\
\hline & Gait & Normal & Normal & Insignificant limp & Significant limp \\
\hline 7. & Strenuous activities & Possible & Limited & Severely limited & Impossible \\
\hline 8. & Radiological Union & Consolidated & Consolidated & Union & Not consolidated \\
\hline
\end{tabular}

\section{Results}

In our study series a some total of 32 patients of segmental tibia fracture treated with intramedullary interlocking nail.

Most of the patients were in age group 40-60 years, with age ranging from $18-65$ years. Out of these 32 cases, 26 were male cases and 6 were female cases.

Expert tibia interlocking nailing system (ETIN) was the implant employed in 10 cases and 22 were treated with intramedullary interlocking nailing system (IMIL).

23 were closed injury and 9 were compound injury, compound injury classification was based on gustilo anderson system.

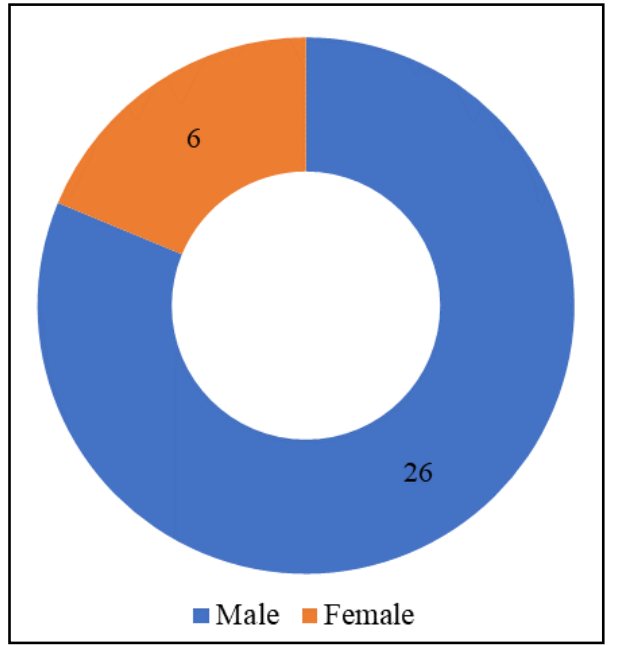

Fig 2: Total cases

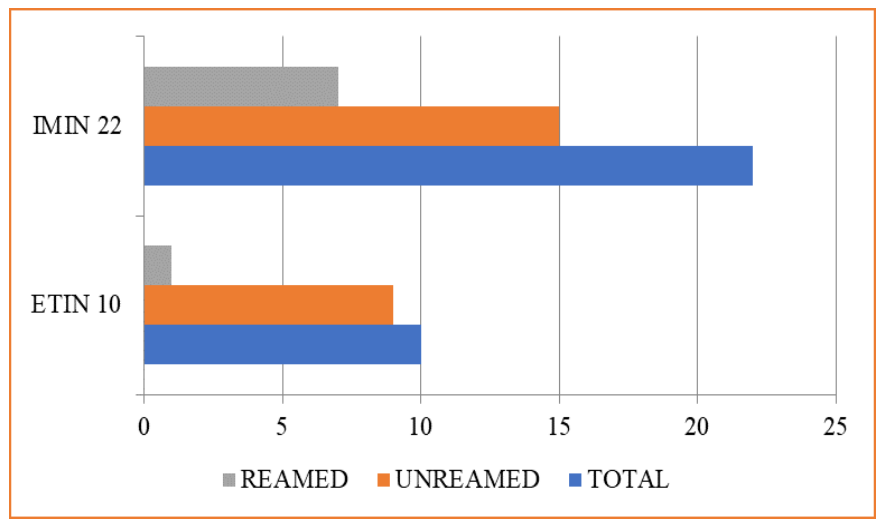

Fig 3: Methods of Intervention

Table 3: Nature of Injury

\begin{tabular}{|c|c|}
\hline Nature of injury & Total \\
\hline Closed & 23 \\
\hline Gustilo Anderson grade 1 & 1 \\
\hline Gustilo Anderson grade 2 & 6 \\
\hline Gustilo Anderson grade 3A & 2 \\
\hline
\end{tabular}

Right side limb involvement was more than left, 19 cases had right side involvement and 13 had left side involvement.

Road traffic accidents was the mode of injury in all the cases. One case had the double segmental tibia fracture, almost all the cases were associated with ipsilateral fibula fracture. Of theses 8 fibula fracture were segmental. None of the cases in study group had fibular fixation. 16 cases were associated with head injury, 8 were associated with clavicle fracture, 2 were associated with rib fracture, one had pneumothorax, ipsilateral metatarsal fracture in 2 cases, bilateral both bone fracture in 1 case.

The interval between injury and surgery ranges from 3-20 days, initial immobilization was with above knee posterior slab in 18 cases and calcaneal pin traction in 14 cases. The mean hospital stay was from 7-30 days, the cases were followed up for 18 months.

The final outcome based on JOHNER AND WRUCH' Scriteria were excellent in 26 cases good in 6 cases.

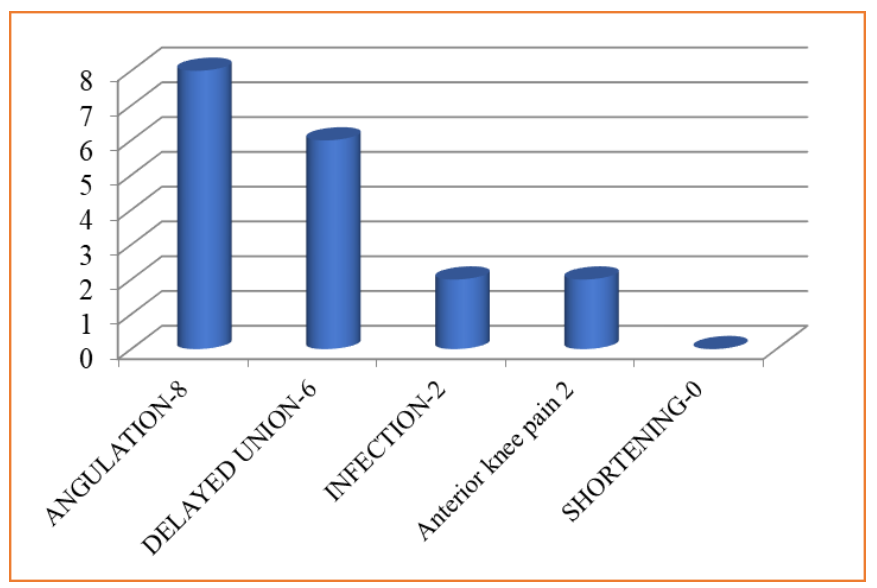

Fig 4: Post-Operative Complication

Most common post operative complication were angulation in 8 cases, angulation less than 10 degrees which was corrected with post operative plaster cast immobilization and 2 cases which had more than 10 degrees angulation were corrected with augmentation plating, 6 cases reported delayed union after waiting for 12 weeks when abundant callus was not visible in the $\mathrm{x}$-ray and the patient had persistent tenderness over the fracture site. These cases were treated with dynamization and bone grafting. One case treated with ETIN had wound infection and delayed union was followed with nail exit, wound debridement and ilizarov fixation as a secondary procedure followed with Regular dressing, antibiotics coverage and guarded weight bearing. One case with gross butterfly fragment angulation was followed with SS wire cerclage. In our series one patient had superficial wound infection which responded to oral antibiotics and daily dressing. 2 cases were followed up with full union and implant exit was done at 18 months. 


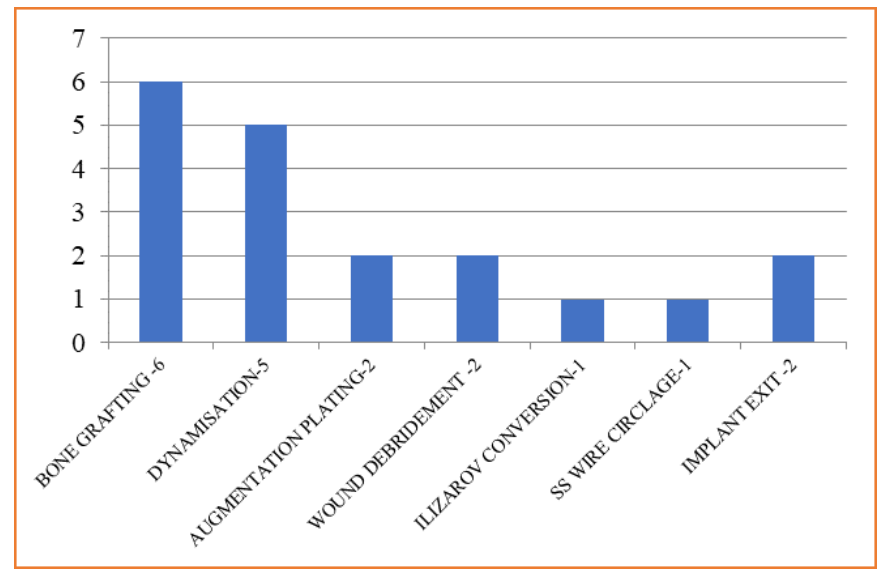

Fig 5: Secondary Procedures

\section{Discussion}

In recent years, the indications for use of the intramedullary nail system were expanded to include the treatment of a wide spectrum of tibial fractures.

The commonest cause of the fracture being high velocity road traffic accidents. In our series all the fractures were due to high velocity road traffic accidents.

Most surgeons advocate treatment of segmental tibia fractures with intramedullary nail placement in light of the significant difficulty and complications rates of other currently available treatment modalities. Intramedullary nail placement avoids many of the concerns related to other modalities such as control of length, rotation, alignment, dissection of the fracture site, disruption of the fracture vascularity, early weight-bearing, and incision site away from traumatic open wounds [9]. Duan et al, in a Cochrane Review on intramedullary nailing for adult diaphyseal tibial fractures, was unable to draw a definitive conclusion on the best technique for intramedullary nail placement although moderate evidence suggests no clear difference in complications between reamed and unreamed nailing. They also noted that reamed nailing demonstrated a decreased incidence of implant failure, less re-operation related to nonunion, but this was only true in closed tibia fractures [11]. In a review of open tibial diaphyseal fractures Mundi et al echoed these findings of superiority of reamed nailing in closed tibia fractures, but no significant difference between methods was detected in open fractures ${ }^{[12]}$.

Initially tibial nails were without interlocking screws, which limited control of the proximal and distal fragments. In 1969 Zucman and Maurer published their treatment of 36 segmental tibial fractures with undreamed Kuntscher-type nails in a blind fashion. Of their 36 patients, 92\% went on to union, but with $16 \%$ rate of septic union all present in patient who sustained open fractures [13]. In 1972 Pantazopoulos et al reported on their results of blind undreamed Kuntscher nailing of 13 segmental tibial fractures with one nonunion, no cases of infection, and no cases of malunion ${ }^{[14]}$. In 1981, Melis et al detailed their treatment of 38 segmental tibia fractures with tight-fitting reamed Kuntscher-Herzog intramedullary nails and supplementation with immobilization in a long leg cast. With strict adherence to this algorithm, in 22 closed and 16 open fractures, they reported one malunion, one non-union, and one infection [9]. Woll and Duwelius reported on their treatment of 31 segmental tibia fractures with seven fractures being treated with undreamed Lottes' nails and the remaining fractures were treated with External fixation, plate osteosynthesis, and nonoperative treatment. Of the four treatment modalities unreamed unlocked Lottes' nails demonstrated the lowest complication rate of $40 \%$ which included malunion and nonunion [6]. The authors were convinced that the high rate of nonunion and malunion was related to the lack of distal rotational control and hypothesized that distally locked intramedullary nails would provide a much lower rate of complications. Merianos et al evaluated the use of Ender nails for the treatment of 22 segmental tibia fractures with all patients achieving union. The limitations of rotation and length control of Ender nails were illustrated as close to one third of fractures were shortened and/or malunited ${ }^{[15]}$.

In 1985 Klemm and Borner detailed one of the early reports of tibial fractures treated between 1976 and 1983 with reamed locked intramedullary nailing. Forty-one of the 401 tibia fractures were segmental with an overall delayed union of $0.8 \%$, infection rate of $2.2 \%$, and an excellent or good outcome in $94 \%$ of patients ${ }^{[16]}$. With the results of Klemm and Birner in mind, $\mathrm{Wu}$ and Shih treated 38 segmental tibial shaft fractures with reamed interlocking intramedullary tibial nailing and achieved a union rate of $97 \%$ without any deep infections, clinically significant malalignment, or implant failures. Of note, all fractures were not immediately treated with intramedullary nailing as a wait time of approximately 1 week was used to allow decreased calf swelling, open wound healing, and stabilization of systemic conditions ${ }^{[17]}$. With the positive results of reamed locked intramedullary nailing of segment tibia fractures, Huang et al treated 33 segmental tibia fractures with this technique. This series detailed rates of $3 \%$ malunion, 9\% delayed unions, no cases of nonunion, and $6 \%$ deep infection rate which occurred in two of the reported open fractures. Giannoudis et al treated 27 cases of segmental tibia fractures with 14 unreamed locking intramedullary nails, 2 reamed locking intramedullary nails, and the remaining 11 cases were treated with other various modalities. There were a large number of complications with 8 of 14 unreamed tibial nails undergoing a secondary procedure to achieve union, eradicate infection, or to undergo amputation. This is in contrast to the 2 reamed locked tibial nails did not undergo secondary procedures or develop complications which could be related to their treatment of only closed fractures ${ }^{[4]}$.

The decision of unreamed versus reamed tibial nails is much less certain in segmental tibia fractures as reports in the literature are exceedingly less common than standard fractures of the tibial diaphysis. One of the main advantages of treatment with an intramedullary nail is the ability to preserve the blood flow to the osseous pathology by minimizing disruption of blood flow from surrounding tissue [10]. The effect of reaming and nail size was evaluated in a canine tibia model with surprising results $[18,19]$. Nutrient artery flow for intact tibias in reamed and unreamed nail placement was evaluated in a mongrel canine model over 14 days post operatively. Blood flow after unreamed nailing went from $44 \%$ of baseline immediately after nailing, but approached baseline with $99 \%$ blood flow at day 14 . This is in stark contrast to the reamed tibial nail with no flow rate at day zero and a resumption of $26 \%$ of baseline at day $14^{[18]}$. Hupel et al used a segmental tibia fracture canine model to evaluate the effect of tibial nail size on osseous cortical blood flow. The canine group with an unreamed "tight" locked intramedullary tibial nail demonstrated a nearly $75 \%$ decrease in blood flow which was still decreased by $50 \%$ at 11 weeks post intervention. Compared to the canine group with an unreamed "loose" locked intramedullary tibial nail which demonstrated an approximately 50\% decrease in blood flow which returned to greater than pre-surgical flow at 11 weeks 
post intervention [19]. One concern with reamed intramedullary nailing of segmental tibia fractures is rotational displacement of the fragments which can strip the surrounding soft tissues and cause an increased rate of avascular complications ${ }^{[20]}$. When using a reamed intramedullary nail it was suggested to reduce and stabilize the fracture with a pointed reduction clamp to avoid this potential stripping of soft tissues from the fracture fragment ${ }^{[4}$, ${ }^{7]}$. With these principals in mind, Kakar and Tornetta followed 51 patients to union with segmental tibia fractures treated with unreamed locked intramedullary nail placement with only a $9 \%$ revision rate ${ }^{[9]}$. Most recently Terra et al compared healing in matched controls of 30 segmental and non segmental tibia fractures treated with 18 unreamed locked intramedullary nails, 4 reamed locked intramedullary nails, 3 plate osteosynthesis, and 5 external fixation. The preferred treatment was unreamed locked intramedullary nailing, but the authors report a greater than $55 \%$ rate of reoperation to obtain union ${ }^{[3]}$. The preferred treatment of closed segmental tibial shaft fractures is reamed locked intramedullary nailing to maximize biomechanical stability of the construct. The preferred treatment of open segmental tibial shaft fractures is unreamed locked intramedullary nailing to maximize fracture biology and to minimize the risk of devascularization of the intercalary segment

The new, multidirectional locked intramedullary system ETIN involves multiple locking options in different planes at the proximal and distal ends. The system has an angular stability locking system that enhances the axial and lateral stability of the fracture fragments. Because of this modified design, the new system has advantages over the traditional tibial intramedullary nail in dealing with both proximal and distal segment tibial fractures. In terms of varus - valgus and flexion - extension, the appendage of oblique screws increased the angular stability. In addition, there are 3 advanced distal locking options in this new tibial intramedullary nail. The set-up involved antero posterior locking option placed very distally, which allowed for optimized bone purchase and prevented damage to the soft tissue. These design modifications ensure that angular stability is retained and angle-stable locking reflects a potential to maintain fixation stability of distal tibia fractures. All of these design modifications allow for better fixation of metaphyseal tibial segments through multiple interlocking holes in close proximity to either end of the nail. The use of multidirectional interlocking screws ensures that alignment can be maintained and that stability can be preserved despite a short proximal segment. This study with a minimum of one and half year follow up duration was taken up at a Tertiary Care Hospital and Research institute-RMMCH in Chidambaram in which the cases were defined as adults having SEGMENTAL tibia fracture (open / close) and all were treated with close reduction and intramedullary tibia nailing.

\section{Case Illustration - 1}

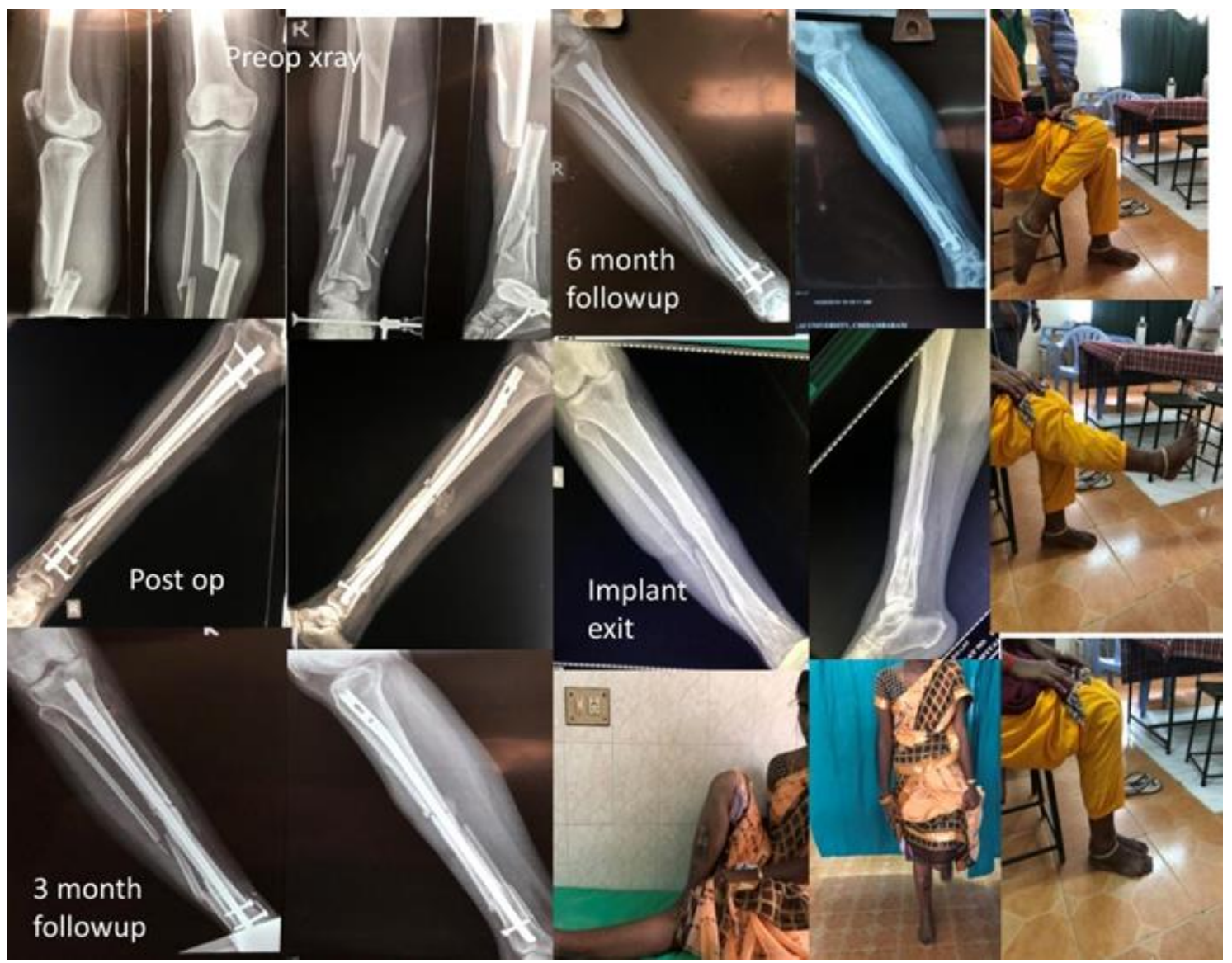




\section{Case Illustration - 2}

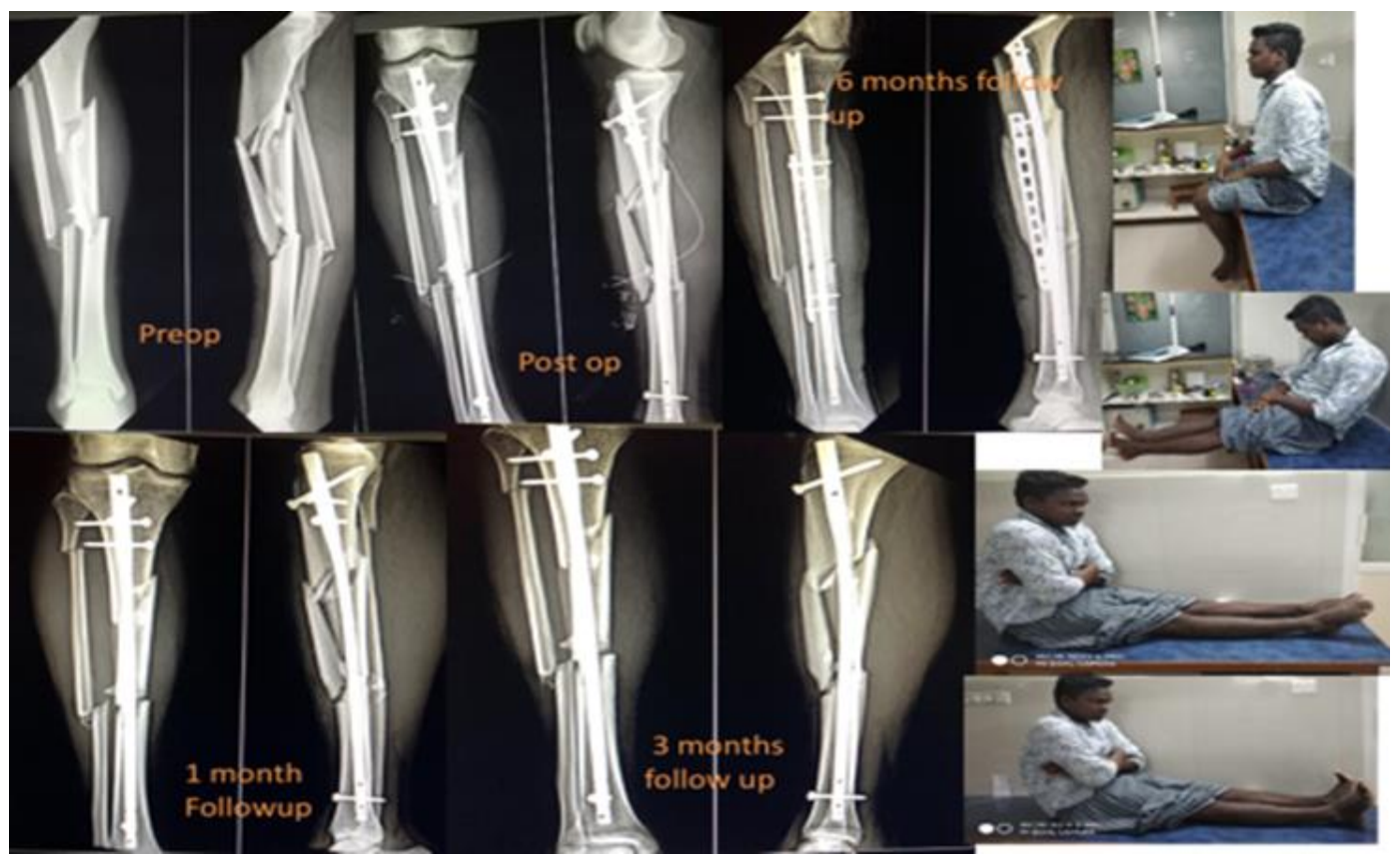

The study started from May 2018 to October 2020. Retrospective cases were taken from the record department of RMMCH. Total 32 cases were taken for the examination during this period. This study is a prospective and retrospective study.

The average intra-op blood loss was $125 \mathrm{ml}$. The patient is positioned supine on a radiolucent table. The ability to flex the knee by at least 90 degree is required. A closed reduction is performed under image intensifier guidance which can be temporarily fixed with reduction clamps. The required nail length is determined using the radiographic ruler. The possibility of axial shortening due to primary compression or secondary dynamisation must be considered when determining the nail length. Depending on the anatomy of the patient and surgical preference, the incision can be trans patellar, medial patellar or even lateral parapatellar and extends from the inferior third of patella to the tibial tuberosity. In our study we use only midline patellar tendon splitting approach. Entry point is crucial in AP view the entry point is in line with the axis of the medullary canal and with the medial aspect of the lateral tubercle of the intercondylar eminence. In the lateral view the entry point is at the ventral edge of the tibial plateau.
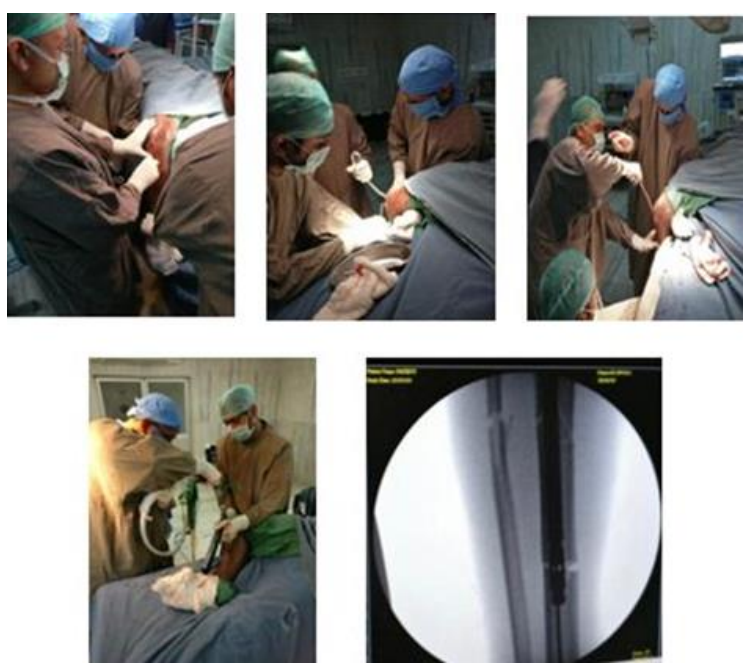

Fig 6: Intra op Images

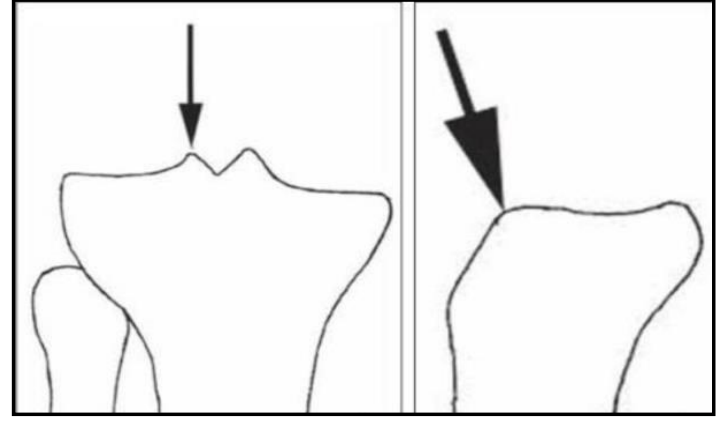

Fig 7: Entry point should be at the medial aspect of laeral tibial spine in AP view and at the ventral edge of tibial plateau in lateral view

Insert the guide wire in line with the medullary canal reaming done when necessary and appropriate nail passed and locking done.the passage of nail through fracture site is closely monitored and confirmed with image intensifier. Confirm proximal and distal nail position in Anteroposterior and lateral views. Consideration must be given for primary compression or secondary dynamisation. Criteria used for acceptable fracture reduction and alignment : >50\% cortical contact; <5$10 \mathrm{deg}$ of varus/valgus angulation when comparing tibial plateau to tibial plafond; $<10-15 \mathrm{deg}$ of anterior or posterior bowing on lateral film; not more than 10-15 mm shortening; < 2-3 deg of internal or external rotation.

We used one or more of the following techniques to reduce the malalignment following nailing of segment tibia having proximal fractures.

1. An ideal entry point in the ventral edge of tibial plateau and in line with the lateral tubercle of the intercondylar eminence.

2. Using femoral distracters/clamps /inter fragmentary screws for reducing the fracture also helps in counteracting the deforming forces.

3. Use of poller screws adjacent to the nail reduces the chance of nail translation and decreases varusvalgus instability.

4. Position: semi-extended .For proximal locking it is recommended to semi-extend the leg in order to relax the muscles.for guide wire passage. limb hanging with knee 
in flexion for reduction by gravity assisted manual reduction.

5. Implant Design: herzog bend and multiplane locking(The expert tibia nail has a Herzog bend which is more distal compared to conventional tibia nail and the angle is 6 degree in the nail used in our study which is less compared to 11 degree in conventional tibia nails that we use)

6. Augmentation plating

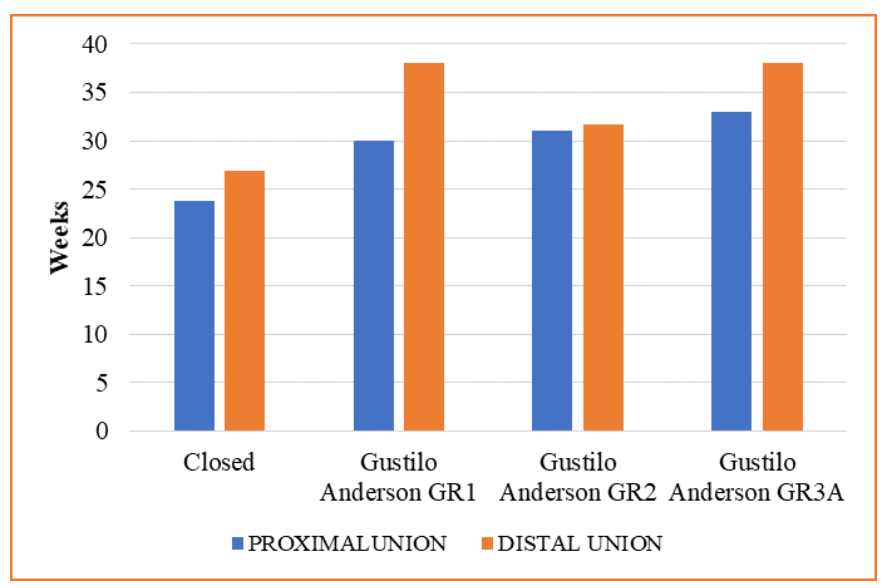

Fig 8: Union in weeks vs nature of injury

In the present study, early weight bearing exercise was started considering fracture pattern, bone and soft tissue condition. As nail being a weight sharing implant, it also helps in preventing regional osteoporosis. Initially partial weightbearing exercise is started. Once it was tolerated by patient, gradually load was increased on fractured limb. Weight bearing exercise is essential for building and maintaining healthy bones. In the present study, when patients begin weight-bearing activities, fracture pattern, fracture localization, the condition of the soft tissue and the quality of the bone should be taken into account. The increase in load is determined according to the fracture segment pattern and localization, the condition of the soft tissue, bone quality as well as the absence or presence of load-induced pain. The concept of dynamization helped in the case of nailing in preventing non-union.

In our study male individuals with age ranging from 40 to 60 years with predominant right limb involvement with history of Road traffic accident have suffered from segmental tibia fractures.

Based on the nature of injury 23 were closed and 9 open injuries. Mean time for union took longer in open cases than closed cases.Open injuries are associated with high velocity of injury causing more soft tissue injury and comminution of segment which are detrimental to bone healing.

Table 4: Nature of Injury

\begin{tabular}{|c|c|c|c|}
\hline Nature of injury & & Proximal union & Distal union \\
\hline Closed & Mean & $23.78 \mathrm{~W}$ & $26.96 \mathrm{~W}$ \\
\hline & SD & 4.552 & 3.612 \\
\hline & N & 23 & 23 \\
\hline Compound & Mean & $31.33 \mathrm{~W}$ & $35.89 \mathrm{~W}$ \\
\hline & SD & 4.63 & 3.01 \\
\hline & N & 9 & 9 \\
\hline
\end{tabular}

Closed segmental fractures without associated complications and minimal displacement were managed with POP -Above knee posterior slab and were taken up for surgery as early as possible with pre surgical work up and anaesthetic concurrence. Open injuries, cases with associated injuries, comminuted and displaced segment fractures were initially managed with calcaneal pin traction and proper wound care.

Gustilo Anderson grade 3 a segmental tibia fracture treated with ETIN with inter fragmentary screwshad delayed union and infection hence wound debridement and nail implant removal done followed with ilizarov fixation as a secondary procedure. with proper wound care and follow up the patient had a good union and range of motion. A patient with Gustilo Anderson classification grade 2 with type 2 diabetes developed superficial infection which was controlled with culture specific oral antibiotics and diabetic control.

Most common postoperative complication is angulation which was reported in eight cases. Angulation of less than $10^{\circ}$ was managed with postoperative POP cast Immobilization. Angulation more than $10^{\circ}$ were corrected by secondary procedure means of augmentation plating.

Delayed union was noted in six cases which was subsequently treated with dynamisation and bone grafting. Shortening less than $1.5 \mathrm{~cm}$ were considered insignificant and not noted as a complication.

Anterior knee pain noted in 2 cases were treated analgesics and physiotherapy. All the cases were done by closed method in primary fixation under $c$ arm guidance. since usage of closed method the surgical scar found to be minimal, anatomical length maintained, and if reduction and rotation aligned to a accepted mannerlimb discrepancy avoided and nailing has better cosmetic advantage. Minimal incision and better scar healing.

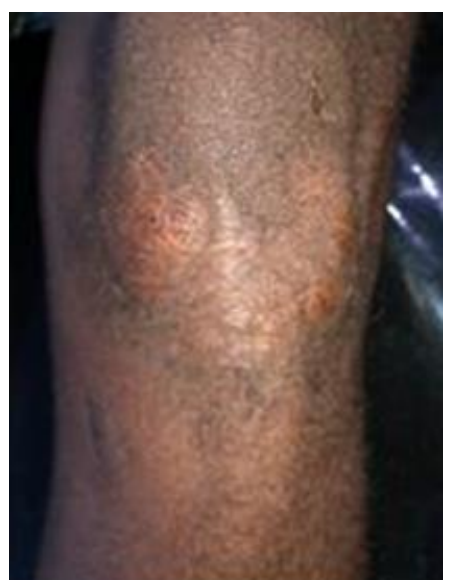

Fig 9: Final surgical scar

The mean time for union was 25.91 weeks for proximal fracture union and 28.88 weeks for distal union.

In our study based on Melis classification of segmental tibia fracture type 1 Melis was found to be more common. All the type 1 Melis fracture were treated with unreamed nailing. Type 4 Melis with long intermediary segment unites faster and better than other types treated with nailing. Reaming was done in 5 cases that to limited to proximal reaming for snugly fit nail passage. Reamed nailing union time was similar to that of non reamed nailing method. 
Table 5: Time of union based melis classification

\begin{tabular}{|c|c|c|c|c|c|c|c|}
\hline Melis & \multicolumn{2}{|c|}{ ETIN } & \multicolumn{2}{c|}{ IMIN } & Proximal union & Distal union & \multirow{2}{*}{ Total } \\
\hline & Reamed & Unreamed & Reamed & Unreamed & Weeks & Weeks & \\
\hline I & 0 & 7 & 0 & 10 & 26.35 & 30.00 & 17 \\
\hline II & 1 & 1 & 1 & 6 & 25.00 & 27.78 & 9 \\
\hline III & 1 & 1 & 1 & 1 & 28.00 & 28.50 & 4 \\
\hline IV & 0 & 0 & 1 & 1 & 22.00 & 25.00 & 2 \\
\hline
\end{tabular}

Proximal fracture in segment tibia unites faster than distal segment tibia fracture.suggesting better muscle coverage near the proximal end helps in healing fracture faster than distal end. We found that union was quicker in the proximal fracture sites than distal, and more in the postero-lateral part of the tibia than the antero-medial part; this may be due to better blood supply and more soft tissue coverage in this area.
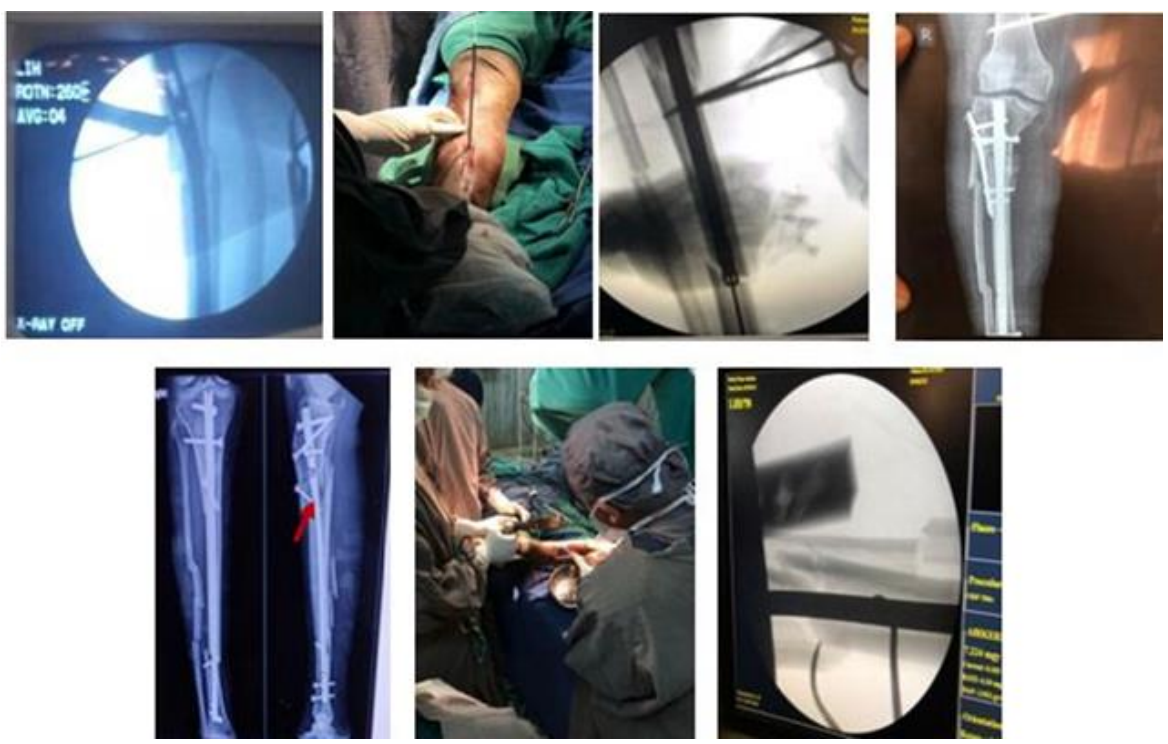

Fig 10: Intra op techniques for reduction

- Segmental fractures are more prone for malalignmentanterior angulation and valgus deformity.

- Proper preoperative planning and specific implant selection needed for reduction

- Reduction of segmental fracture involves various techniques like poller screws, joystick manoeuvre, distractors, Buttress plate, interfragmentary screws.

- Malalignment which are particular for proximal segment tibial fractures could not be avoided completely in all cases but postoperative timely intervention had resulted in excellent to good final outcome.

Closed reduction and internal fixation with Tibia nailing is a very effective method in surgical treatment of segmental tibial fractures.

Patient counselling regarding the complex nature of injury and the possible requirement of secondary procedures should be anticipated and addressed. Thorough post operative rehabilitation may be needed in segmental fractures. The patient should be informed early of this possibility.

New radiological criteria for bone healing should be defined for these fractures due to the longer time to cure. Compromised blood supply to the intermediary segment and to both segments, requires an interventional approach if osteogenesis lacks vigor.

The interval between injury and surgery ranges from 3-20 days. The mean hospital stay was from 7-30 days. Patients operated with this technique can be ambulated early without external immobilization in majority of cases, patients are allowed to resume work early as tolerated and this procedure also reduces the hospital stay and boosts up the morale of the patient.

\section{Conclusion}

Patient counselling regarding the complex nature of injury and the possible requirement of secondary procedures should be anticipated and addressed.The results of our study suggest that if soft-tissue handling and restoration of anatomical alignment are successful in the treatment of challenging segmental tibial fractures, IMN will be good treatment method.

\section{References}

1. Mcmurtry J, Mounasamy V. Segmental Tibia Fractures. Ann Orthop Rheumatol 2015;3(3):1051.

2. Langård $\mathrm{O}, \mathrm{Bo} \mathrm{O}$. Segmental tibial shaft fractures. Acta Orthop Scand 1976;47:351-357.

3. Teraa M, Blokhuis TJ, Tang L, Leenen LP. Segmental tibial fractures: an infrequent but demanding injury. Clin Orthop Relat Res 2013;471:2790-2796.

4. Giannoudis PV, Hinsche AF, Cohen A, Macdonald DA, Matthews SJ, Smith RM. Segmental tibial fractures: an assessment of procedures in 27 cases. Injury 2003;34:756-762.

5. Rommens PM, Coosemans W, Broos PL. The difficult healing of segmental fractures of the tibial shaft. Arch Orthop Trauma Surg 1989;108:238-242.

6. Woll TS, Duwelius PJ. The segmental tibial fracture. Clin Orthop Relat Res 1992, P204-207.

7. Melis GC, Sotgiu F, Lepori M, Guido P. Intramedullary nailing in segmental tibial fractures. J Bone Joint Surg Am 1981;63:1310-1318.

8. Sarmiento A, Latta LL. Functional treatment of closed segmental fractures of the tibia. Acta Chi Orthop Traumatol Cech 2008;75:325-331. 
9. Kakar S, Tornetta P 3rd. Segmental tibia fractures: a prospective evaluation. Clin Orthop Relat Res 2007;460:196-201.

10. Oztürkmen Y, Karamehmetoğlu M, Karadeniz H, Azboy I, Caniklioğlu M. Acute treatment of segmental tibial fractures with the Ilizarov method. Injury 2009;40:321326.

11. Duan X, Al-Qwbani M, Zeng Y, Zhang W, Xiang Z. Intramedullary nailing for tibial shaft fractures in adults. Cochrane Database Syst Rev 2012.

12. Mundi R, Chaudhry H, Niroopan G, Petrisor B, Bhandari, M. Open Tibial Fractures: Updated Guidelines for Management. JBJS Reviews 2015, 3.

13. Zucman J, Maurer P. Two-level fractures of the tibia. Results in thirtysix cases treated by blind nailing. J Bone Joint Surg Br 1969;51:686-693.

14. Pantazopoulos T, Galanos P, Agoropoulos Z, Hartofilakidis - Garofalidi. Treatment of double tibial fractures by blind intramedullarynailing. Clin Orthop Relat Res 1972;84:137-143.

15. Merianos P, Papagiannakos K, Scretas E, Smyrnis P. Ender nails for segmental tibial fracture. Early weight bearing in 22 cases. Acta Orthop Scand 1988;59:297-301.

16. Klemm KW, Börner M. Interlocking nailing of complex fractures of the femur and tibia. Clin Orthop Relat Res 1986, P89-100.

17. Wu CC, Shih CH. Segmental tibial shaft fractures treated with interlocking nailing. J Orthop Trauma 1993;7:468472.

18. Brinker MR, Cook SD, Dunlap JN, Christakis P, Elliott MN. Early changes in nutrient artery blood flow following tibial nailing with and without reaming: a preliminary study. J Orthop Trauma 1999;13:129-133.

19. Hupel TM, Aksenov SA, Schemitsch EH. Cortical bone blood flow in loose and tight fitting locked undreamed intramedullary nailing: a canine segmental tibia fracture model. J Orthop Trauma 1998;12:127-135.

20. Arastu MH, Sheehan B, Paolucci EO, Buckley RE. Does it really spin? Intra-medullary nailing of segmental tibial fractures--a cadaveric study. Injury 2015;46:643-648. 